\title{
Diagnostic value of miR-30d-5p and miR-125b-5p in acute myocardial infarction
}

\author{
KEGANG JIA ${ }^{1}$, PING SHI ${ }^{1}$, XUEJING HAN ${ }^{1}$, TIENAN CHEN $^{2}$, HONGXIA TANG ${ }^{1}$ and JING WANG ${ }^{1}$ \\ Departments of ${ }^{1}$ Clinical Laboratory and ${ }^{2}$ Cardiovascular Surgery, \\ TEDA International Cardiovascular Hospital, Tianjin 300457, P.R. China
}

Received August 3, 2015; Accepted February 2, 2016

DOI: $10.3892 / \mathrm{mmr} .2016 .5246$

\begin{abstract}
Rapid and accurate differential diagnosis of acute myocardial infarction (AMI) is crucial for timely interventions and the improvement of prognosis. However, this is difficult to achieve using current methods. Therefore, the present study aimed to evaluate the suitability of circulating microRNAs (miRNAs) as AMI biomarkers in patients with acute coronary syndrome (ACS). miRNA profiling in plasma samples from patients with AMI $(n=3)$ and healthy controls $(n=3)$ was performed using microarrays. Results were then validated in five patients and five healthy controls. miRNA-125b-5p and miR-30d-5p expression levels were quantified in plasma samples from 230 patients with ACS and 79 healthy controls using reverse transcription-quantitative polymerase chain reaction. Routine diagnostic parameters were assessed, including creatinine kinase MB, cardiac troponin I (cTnI) and myoglobin. A total of 33 miRNAs were differentially expressed in patients with AMI and healthy controls. Following validation based on the previously established roles for these miRNAs, six miRNAs were validated. miR-125b-5p and miR-30d-5p were selected for further investigation. Expression levels of miR-125b-5p and miR-30d-5p in plasma were higher in patients with ACS compared with the healthy controls $(\mathrm{P}<0.001)$. Receiver operating characteristic curve analysis revealed that the area under the curve of miR-30d-5p was higher than that of cTnI (0.915 and 0.899). miR-125b-5p (sensitivity, 0.808; specificity, 0.845) and miR-30d-5p (sensitivity, 0.855; specificity, 0.810) were suitable diagnostic predictors of AMI. Kaplan-Meier survival analysis indicated that miR-125b-5p levels were associated with 6 month cardiovascular events in patients with AMI, but not miR-30d-5p. miR-125b-5p and miR-30d-5p presented a diagnostic value for early diagnosis of AMI, and miR-30d-5p may have a higher diagnostic value than cTnI.
\end{abstract}

Correspondence to: Dr Kegang Jia, Department of Clinical Laboratory, TEDA International Cardiovascular Hospital, 61 Third Road, Tianjin 300457, P.R. China

E-mail:txjia@126.com

Key words: acute myocardial infarction, miR-125b-5p, miR-30d-5p, prognosis, diagnosis, microarrays

\section{Introduction}

Acute coronary syndrome (ACS) covers the spectrum of acute myocardial ischemia and/or necrosis that is commonly secondary to reduced coronary blood flow. ACS also includes unstable angina pectoris (UAP) and acute myocardial infarction (AMI) (1-5). ACS is common in emergency rooms and each year it is responsible for 1 million hospital admissions in the USA and 2 million in Europe (5). Early diagnosis and intervention are crucial in order to minimize the damage to the cardiac muscle (1-5), as treatment may significantly reduce mortality and improve long-term prognosis (6). For accurate ACS diagnosis, physical examination, electrocardiography, radiologic studies and cardiac biomarker tests are essential, and these also aid in guiding treatment (1). Timely and appropriate treatment for ACS includes cardiac catheterization and primary percutaneous coronary intervention (7).

Since clinical presentation and echocardiography findings are often nonspecific in patients with chest pain, cardiac biomarkers, including cardiac troponin I (cTnI), creatine kinase MB (CK-MB) mass and myoglobin (Myo) are often used for diagnosis $(8,9)$. Indeed, $\mathrm{cTnI}$ is the gold standard for diagnosis of AMI (10), however, serial testing is required as it is also frequently present in patients with chronic but stable coronary artery disease and may be detected in apparently healthy controls $(11,12)$. In addition, the timing of troponin measurement with respect to symptom onset has an impact on the result $(13,14)$. Therefore, novel biomarkers with high sensitivity and specificity for early diagnosis of AMI are urgently required to improve the prognosis of patients with acute chest pain.

Notably, the role of microRNAs (miRNAs) in acute myocardial infarction (AMI) has been previously investigated $(15,16)$. Levels of muscle-specific miR-1, miR-133a and miR-499 in addition to cardiac-specific miR-208a were significantly higher in plasma samples from patients with AMI compared with controls $(16,17)$. Circulating miRNAs are readily detectable, relatively stable and tissue-specific (14), making them attractive biomarker candidates.

It was hypothesized that specific miRNAs may be associated with AMI. The aim of the present study was to comprehensively assess the miRNAs released into circulation during AMI, and determine which may be used as biomarkers to detect and monitor myocardial injury. In addition, miRNA 
Table I. Primers used for reverse transcription-quantitative polymerase chain reaction.

\begin{tabular}{lll}
\hline microRNAs & \multicolumn{1}{c}{ Forward $\left(5^{\prime}-3^{\prime}\right)$} & Reverse $\left(5^{\prime}-3^{\prime}\right)$ \\
\hline hsa-miR-125b-5p & GCTCCCTGAGACCCTAAC & GTGCGTGTCGTGGAGTCG \\
hsa-miR-136-5p & GGAACTCCATTTGTTTTGA & CAGTGCGTGTCGTGGAGT \\
hsa-miR-129-1-3p & GAAGCCCTTACCCCAAA & CAGTGCGTGTCGTGGA \\
hsa-miR-30d-5p & GGTGTAAACATCCCCGAC & CAGTGCGTGTCGTGGAG \\
hsa-miR-27a-5p & GGAGGGCTTAGCTGCTTGT & GTGCGTGTCGTGGAGTCG \\
hsa-miR-1291 & TCGCCCTGACTGAAGACC & CAGTGCGTGTCGTGGAGT
\end{tabular}

expression levels were compared with established biomarkers, including CK-MB, Myo and cTnI.

\section{Patients and methods}

Study design and patients. Circulating miRNAs were profiled in 3 patients with AMI and 3 healthy controls. These results were then validated by profiling the same miRNAs in 5 additional patients with AMI and healthy controls. The selected miRNAs were further assessed with a larger sample size, including 230 consecutive patients with ACS and 79 healthy controls (normal electrocardiograms and no history of cardiovascular diseases). Exclusion criteria were as follows: i) Presence of chest pain for $>3 \mathrm{~h}$ at admission; or ii) angiography was not performed.

Plasma samples from patients were collected at the TEDA International Cardiovascular Hospital Emergency Department (Tianjin, China) between September 2011 and September 2013. Serial blood samples were collected from individuals with AMI at 0-3, 3-6, 6-9, 9-12 and 12-24 h following admission. Diagnoses of ACS, UA, AMI, ST-elevated myocardial infarction (STEMI) and non-ST-elevated myocardial infarction (NSTEMI) were made according to international standards (11-13). All participants underwent clinical evaluation, including physical examination, 12-lead electrocardiography and echocardiography examinations. Blind diagnoses were made by two independent experienced cardiologists unaware of the miR-30d-5p and miR-125b-5p data. Demographic and diagnosis information was collected for each patient, including age, gender, coronary risk factors (hypertension, diabetes mellitus, hyperlipidemia and smoking), renal function and duration of chest pain. The present study was approved by the TEDA International Cardiovascular Hospital Ethics Committee (Tianjin, China), and written informed consent was obtained from each individual.

Sample preparation. Peripheral blood was centrifuged at $820 \mathrm{x} \mathrm{g}$ for $5 \mathrm{~min}$ at room temperature, and the resulting plasma samples were transferred into new RNase/DNase-free Eppendorf tubes (Thermo Fisher Scientific, Inc., Waltham, MA, USA), and stored at $-80^{\circ} \mathrm{C}$ until RNA extraction. Total plasma RNA was isolated and eluted in $100 \mu \mathrm{l}$ RNase-free water using a mirVana PARIS kit (\#1556, Ambion; Thermo Fisher Scientific, Inc.), according to the manufacturer's protocol.

miRNA array analysis. In order to assess the differential miRNA expression in patients with AMI, miRNA expression profiling was performed with plasma samples from patients with AMI $(n=3)$ and healthy controls $(n=3)$ using the miRCURY LNA microRNA Array system (version 18.0; Exiqon Inc., Woburn, MA, USA). Total RNA was prepared using TRIzol (Invitrogen; Thermo Fisher Scientific, Waltham, MA, USA) and an miRNeasy Mini kit (Qiagen GmbH, Venlo, Netherlands) according to the manufacturers' protocol. RNA integrity was assessed by electrophoresis on a $1.2 \%$ denaturing agarose gel at $120 \mathrm{~V}$ for $15 \mathrm{~min}$. Intact total RNA was characterized by sharp 28S and 18S rRNA bands (eukaryotic samples). All plasma RNA preparations were quantified on a NanoDrop 1000 spectrophotometer (Thermo Fisher Scientific, Inc.). Samples with absorbance (260/280) ratios $>1.8$ were considered to be optimal for microarray assays. RNA samples were labeled with the miRCURY Hy3 Power labeling kit (Exiqon, Vedbaek, Denmark) and hybridized on the miRCURY LNA microRNA Array system. The slides were washed three times using a wash buffer kit (Exiqon), then dried by centrifugation at $1.5 \mathrm{x} \mathrm{g}$, for $5 \mathrm{~min}$ at room temperature. Next, the slides were scanned on an Axon GenePix 4000B microarray scanner (Molecular Devices, LLC, Sunnyvale, CA, USA). Scanned images were imported into the GenePix Pro software (version 6.0; Molecular Devices, LLC) for grid alignment and data extraction. Replicated miRNAs were averaged and miRNAs with intensities $\geq 30$ in all samples were selected for calculating the normalization factor. Data were normalized using the Median normalization (18). Significantly differentially expressed miRNAs were identified through Volcano Plot filtering. Hierarchical clustering was also performed to indicate the various miRNA expression profiling among samples. The threshold value for significance of miRNA upregulation or downregulation was set at fold-change $\geq 2.0$, with $\mathrm{P}<0.05$ calculated by Student's t-test. The miRNAs selected for investigation in the current study were further filtered based on their expression levels, described in previously published data, and their heart-specificity defined according to umm.uni-heidelberg.deapps/zmf/mirwalk/disease.php (19).

Reverse transcription-quantitative polymerase chain reaction (RT-qPCR). Total RNA (5 $\mu \mathrm{l})$ was reverse-transcribed using the TaqMan microRNA Reverse Transcription kit (Applied Biosystems; Thermo Fisher Scientific, Inc.) according to the manufacturer's protocol. Prior to RT, RNA was eluted in nuclease-free water in order to avoid DNA contamination. Temperatures used for RT were $16^{\circ} \mathrm{C}$ for $30 \mathrm{~min}, 42^{\circ} \mathrm{C}$ for $30 \mathrm{~min}$, and $85^{\circ} \mathrm{C}$ for $5 \mathrm{~min}$. The primer sequences used were obtained from Invitrogen (Thermo Fisher Scientific, Inc.) and 
Table II. MicroRNAs were differentially expressed in patients with AMI compared with healthy controls by microarrays.

\section{A, Upregulated}

\begin{tabular}{|c|c|c|}
\hline microRNAs & Fold-change & P-value \\
\hline has-miR-24-3p & 7.563 & 0.034 \\
\hline has-miR-29a-5p & 7.615 & 0.005 \\
\hline has-miR-125b-5p & 40.002 & 0.004 \\
\hline hsa-let-7b-5p & 16.004 & 0.008 \\
\hline hsa-let-7g-5p & 5.058 & 0.038 \\
\hline hsa-miR-125a-5p & 12.485 & 0.002 \\
\hline hsa-miR-149-5p & 6.772 & 0.035 \\
\hline hsa-miR-330-5p & 9.886 & 0.004 \\
\hline hsa-miR-101-3p & 9.706 & 0.024 \\
\hline hsa-miR-34c-5p & 2.325 & 0.048 \\
\hline hsa-miR-139-5p & 39.982 & 0.028 \\
\hline hsa-miR-29b-1-5p & 2.179 & 0.034 \\
\hline hsa-miR-132-5p & 10.184 & 0.023 \\
\hline hsa-miR-212-3p & 4.204 & 0.021 \\
\hline hsa-miR-129-1-3p & 16.264 & 0.003 \\
\hline hsa-miR-138-5p & 249.663 & 0.010 \\
\hline hsa-miR-9-5p & 31.712 & 0.016 \\
\hline hsa-miR-30d-5p & 6.233 & 0.026 \\
\hline hsa-miR-29c-5p & 6.756 & 0.038 \\
\hline hsa-miR-138-2-3p & 3.074 & 0.010 \\
\hline hsa-miR-129-5p & 21.802 & 0.024 \\
\hline hsa-miR-124-3p & 301.144 & 0.027 \\
\hline hsa-let-7i-5p & 10.096 & 0.002 \\
\hline hsa-miR-22-3p & 9.958 & 0.043 \\
\hline hsa-miR-9-3p & 16.504 & 0.020 \\
\hline hsa-miR-136-5p & 448.976 & 0.011 \\
\hline hsa-miR-15a-3p & 3.325 & 0.015 \\
\hline
\end{tabular}

B, Downregulated

\begin{tabular}{lcc}
\hline microRNAs & Fold-change & P-value \\
\hline hsa-miR-500a-5p & 0.385 & 0.047 \\
hsa-miR-27a-5p & 0.046 & 0.012 \\
hsa-miR-142-5p & 0.200 & 0.027 \\
hsa-miR-664a-5p & 0.212 & 0.020 \\
hsa-miR-1246 & 0.173 & 0.046 \\
hsa-miR-1291 & 0.033 & 0.116
\end{tabular}

The threshold value for significance used for miRNA upregulation or downregulation was set at fold change $\geq 2.0$ with a $\mathrm{P}<0.05$ calculated by t-test. All differentially expressed microRNAs are available upon request.

are described in Table I. Subsequently, $2.33 \mu 1$ cDNA was used to assess miRNA expression by qPCR using the TaqMan microRNA Assay kits (hsa-miR-125b-5p, assay id.477885_mir; hsa-miR-136-5p, assay id. 478307_mir; hsa-miR-129-1-3p, assay id. 480873_mir; hsa-miR-30d-5p, assay id. 478606_mir;
hsa-miR-27a-5p, assay id. 477998_mir; hsa-miR-1291, assay id. 478690_mir; cel-miR-39-3p, assay id. 478293_mir; Applied Biosystems; Thermo Fisher Scientific, Inc.) on a Light Cycler 480 Real Time PCR System (Roche Diagnostics, Basel, Switzerland). All reactions involved an initial denaturation step at $95^{\circ} \mathrm{C}$ for $10 \mathrm{~min}$ followed by 40 cycles of $95^{\circ} \mathrm{C}$ for $15 \mathrm{sec}$, and $60^{\circ} \mathrm{C}$ for $60 \mathrm{sec}$. The $\mathrm{C}_{\mathrm{q}}$ value was defined as the number of PCR cycles required for the fluorescence signal to exceed the detection threshold value (20). Values were normalized by spiking in $5 \mathrm{fmol} / \mu \mathrm{l}$ cel-miR-39-3p (Invitrogen; Thermo Fisher Scientific, Inc.) in the patient samples (21) from the cel-miR-39-3p TaqMan MicroRNA Assay kit and ratios were derived as previously proposed (22).

Biochemical analyses. Peripheral blood was collected in tubes containing EDTA and centrifuged at $820 \mathrm{x} \mathrm{g}$ for $5 \mathrm{~min}$ at room temperature. cTnI and Myo levels were determined in plasma samples by chemiluminescence immunoassays, using the accuTnI and Access Myoglobin kits respectively from Beckman Coulter, Inc. (Brea, CA, USA). CK-MB was assessed by a quantitative mass assay using the Access CK-MB kit (Beckman Coulter, Inc.).

Study endpoint and follow-up. The study endpoint was defined as the occurrence of cardiovascular death, myocardial infarction, hospitalization for unstable angina, stroke, coronary revascularization procedures, peripheral revascularization procedures or heart failure requiring hospitalization (23). To avoid multiple counting of patients with more than one event, each patient contributed only once to the composite endpoint. Endpoints or events were determined by reviewing the medical records or by follow-up telephone interviews for up to 12 months following the initial chest pain episode.

Statistical analysis. Statistical analysis was performed using SPSS (version 13.0; SPSS, Inc., Chicago, IL, USA) and GraphPad Prism (version 5.0; GraphPad Software, Inc., San Diego, CA, USA). The normality of the data was assessed using Shapiro-Wilks test or Kolmogorov-Smirnov test. Normally distributed data is expressed as the mean \pm standard error, and the differences between groups were compared by one-way analysis of variance with Tukey's honest significant difference test used for post-hoc analysis or independent samples t-test. The normally distributed data has been indicated in Table III. Data that was not normally distributed was expressed as the median (min, max) or median and interquartile range, and the differences between groups were compared using the non-parametric Kruskal-Wallis one-way analysis of variance. The differences between qualitative categorical data groups were compared using the Pearson's $\chi^{2}$ test. Receiver operating characteristic (ROC) curves were established for discriminating AMI. Each cardiac biomarker was examined, and ROC curves and optimal cut-off values were obtained. In addition, the sensitivity, specificity, positive predictive value and negative predictive value of the candidate biomarkers were determined. The correlations of cardiac biomarkers with end point events at 1-, 6-and 12-month follow-ups were evaluated by the Kaplan-Meier, log-rank and Cox regression tests. Kaplan-Meier was used to draw the Kaplan-Meier curves, and log-rank tests were used to detect whether there were 


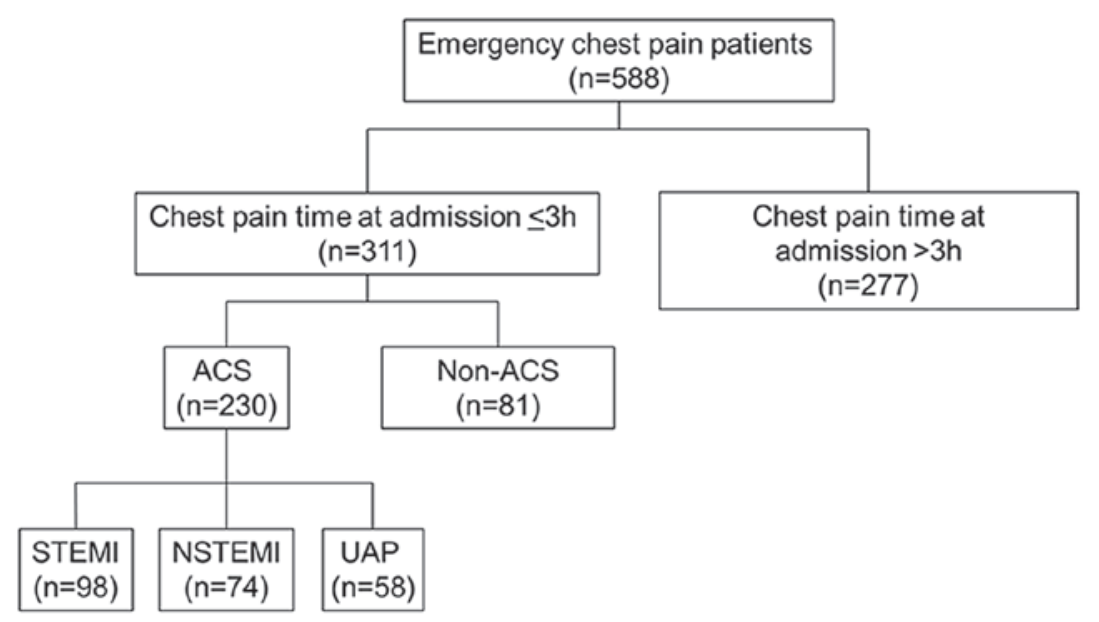

Figure 1. Patient flowchart. ACS, acute coronary syndrome; STEMI, ST-elevated myocardial infarction; NSTEMI, non-ST-elevated myocardial infarction; UAP, unstable angina pectoris.

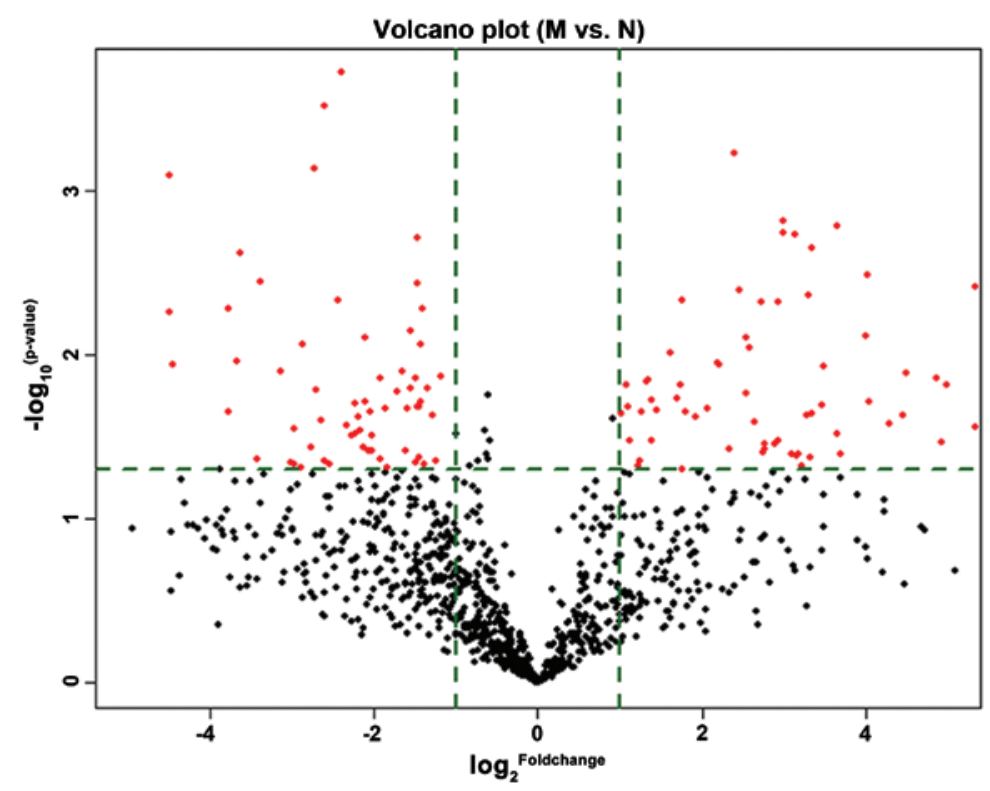

Figure 2. Volcano plots constructed using fold-change and P-values, visualizing the relationship between fold-change (magnitude of change) and statistical significance (which takes both magnitude of change and variability into consideration). The dashed vertical lines correspond to 2.0 -fold up and down, respectively, and the horizontal line represents a P-value of 0.05 . Therefore, the red points in the plot are the significantly differentially expressed miRNAs.

significant differences between the Kaplan-Meier curves. Cox regression analysis was performed to assess the risk factors involved in the prognosis of patients with ACS Two-tailed $\mathrm{P}<0.05$ was considered to indicate a statistically significant difference.

\section{Results}

Patients and controls. Profiling of the circulating miRNAs was performed in 3 patients with AMI and 3 healthy controls. The results were then assessed in a validation cohort of additional patients with AMI $(n=5)$ and healthy controls $(n=5)$. Next, the study was extended to a larger sample size (patients, $n=230$; controls, $n=79$ ). Fig. 1 presents the patients' flowchart. Among 588 patients who were admitted for chest pain, 277 were excluded, as the time between pain onset and admission was $>3 \mathrm{~h}$; and 81 were excluded due to the cause of chest pain not being ACS. Therefore, 230 patients were included (98 with STEMI, 74 with NSTEMI and 58 with UAP). A total of 79 healthy controls were recruited.

Detection of circulating miRNAs by microarrays. A total of 33 miRNAs were differentially expressed in patients with AMI $(n=3)$ and healthy controls $(n=3)$ (Table II). Hierarchical clustering for the 33 differentially expressed miRNAs is indicated by a volcano plot (Fig. 2). Fig. 3 represents a heat map of these miRNAs. As indicated by Table II, a total of 27 miRNAs were upregulated and 6 were downregulated in patients with AMI compared with healthy controls.

Circulating miRNAs in the validation cohort. Among the 33 significantly differentially expressed miRNAs in patients with AMI $(n=3)$ and healthy controls $(n=3), 4$ upregulated (miR-125b-5p, miR-30d-5p, miR-136-5p, miR-129-1-3p) and 


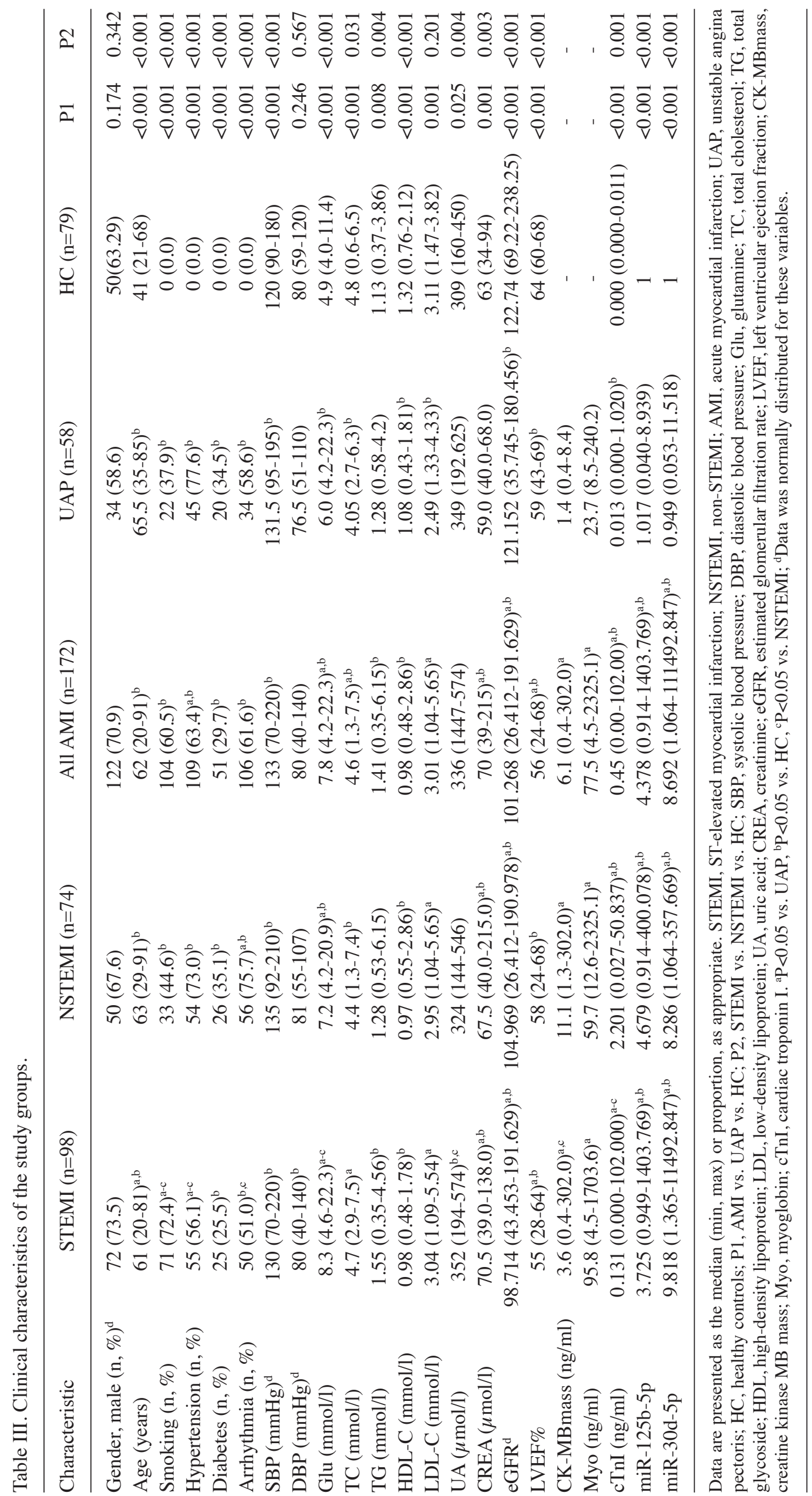




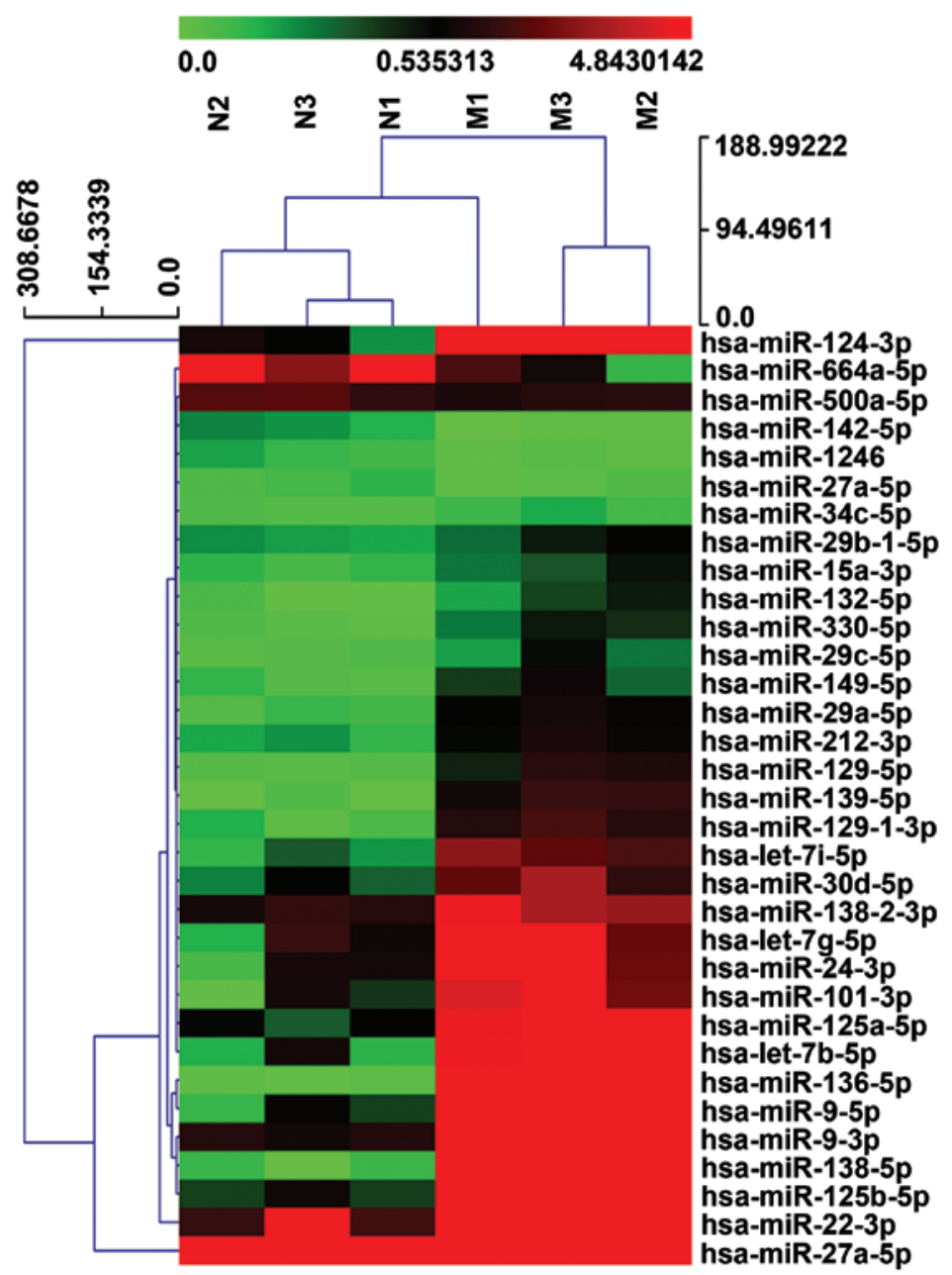

Figure 3. Profiling of circulating miRNAs in patients with AMI and healthy controls. RNA was isolated from the plasma of healthy controls (N1, N2, N3) and AMI patients (M1, M2, M3). The heat map diagram represents the clustering of the 33 differentially expressed miRNAs. Red indicates higher expression, and green low expression. Data is summarized in Table II. AMI, acute myocardial infarction; M1, AMI patient 1; M2, AMI patient 2; M3, AMI patient 3; $\mathrm{N} 1$, healthy control 1; N2, healthy control 2; N3, healthy control 3.

A

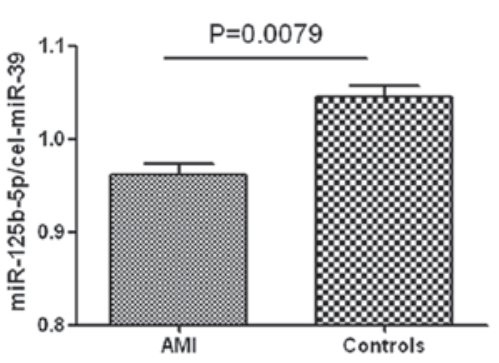

D

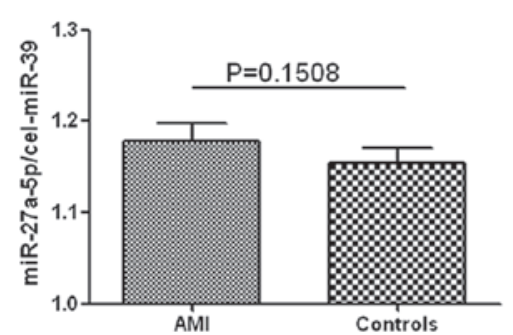

B

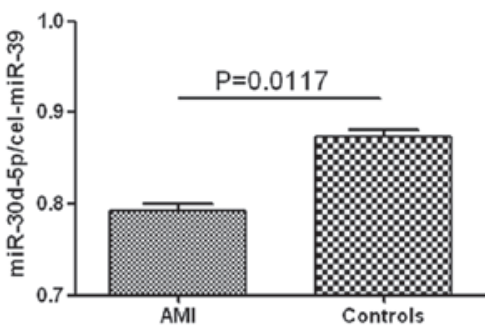

$\mathbf{E}$

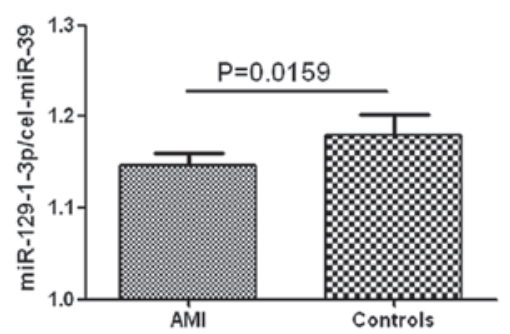

C

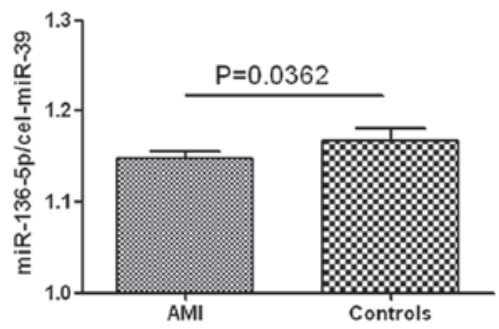

F

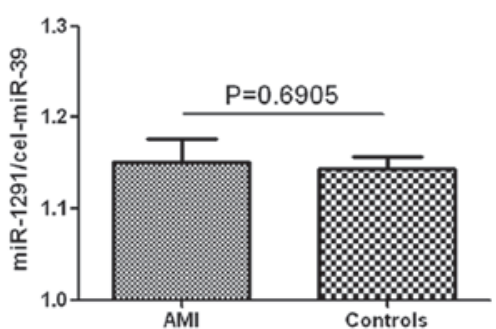

Figure 4. Circulating (A) miR-125b-5p, (B) miR-30d-5p, (C) miR-136-5p, (D) miR-27a-5p, (E) miR-129-1-3p and (F) miR-1291 expression levels in the validation cohort. MicroRNAs levels were determined by reverse transcription-quantitative polymerase chain reaction. Values were normalized to cel-miR-39 and ratios obtained by the $2^{-\Delta \Delta C q}$ method. Data are presented as the mean \pm standard error ( $\mathrm{n}=5$ each group). AMI, acute myocardial infarction. 
A
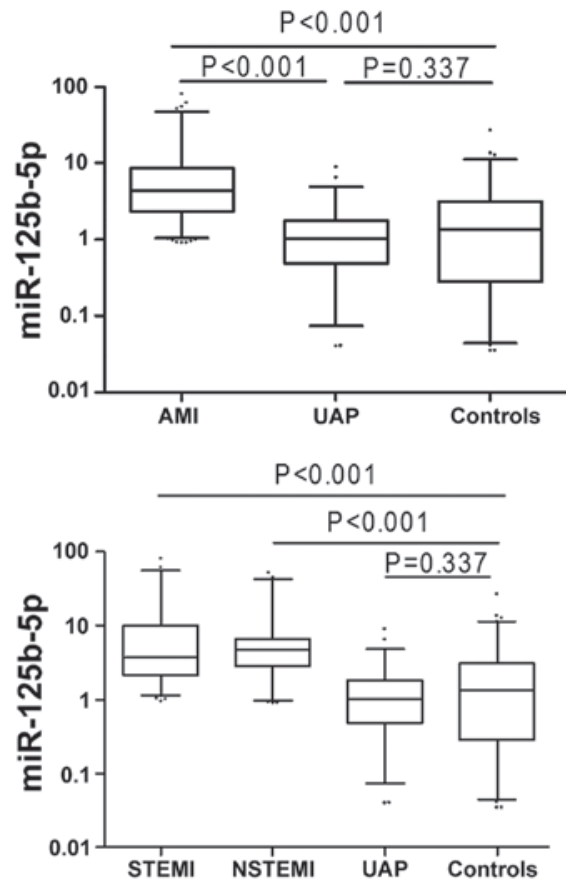

B
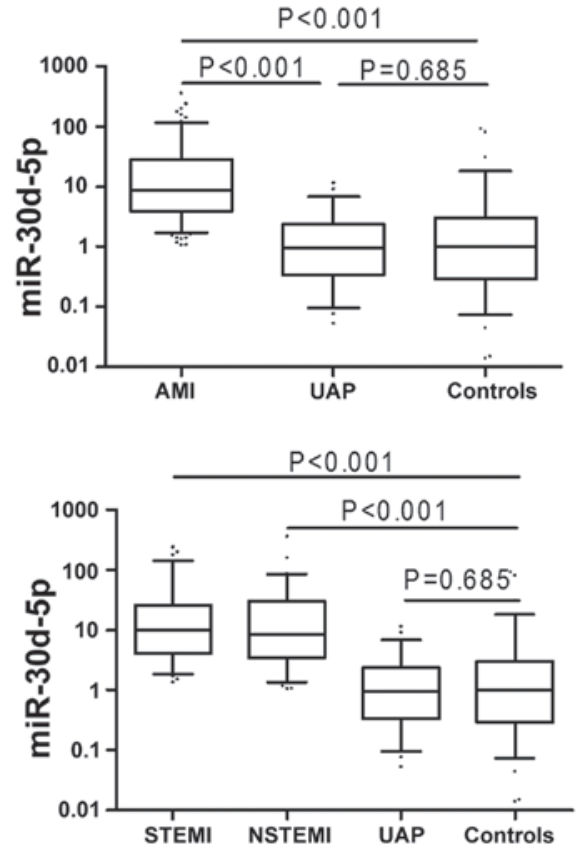

Figure 5. Expression levels of (A) miR-125b-5p and (B) miR-30d-5p in plasma samples of patients with AMI, UAP, STEMI, NSTEMI and healthy control groups. Expression levels were determined by reverse transcription-quantitative polymerase chain reaction. Data are presented as the median and interquartile range. AMI, acute myocardial infarction; UAP, unstable angina pectoris; STEMI, ST-elevated myocardial infarction; NSTEMI, non-ST-elevated myocardial infarction.

2 downregulated (miR-27a-5p and miR-1291) miRNAs were selected as targets for further investigation based on previously published data $(14,23,24)$. Their expression levels in samples from patients with AMI $(\mathrm{n}=5)$ and healthy controls $(n=5)$ were determined by RT-qPCR, with cel-miR-39 used as an internal control. As indicated in Fig. 4, fold-changes of miR-125b-5p, miR-30d-5p, miR-136-5p and miR-129-1-3p were $4.46(\mathrm{P}=0.008), 4.29(\mathrm{P}=0.012), 1.42(\mathrm{P}=0.036)$ and $1.75(\mathrm{P}=0.016)$ while miR-27a-5p and miR-1291 were

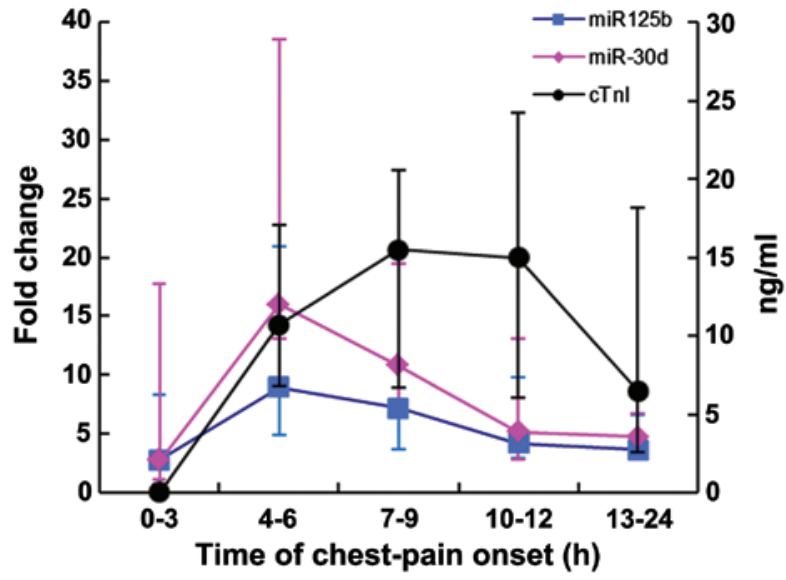

Figure 6. Fold-changes of miR-125b-5p, miR-30d-5p and cTnI in 20 patients with AMI at different time points following the onset of chest pain. Data are presented as the median $(\min , \max )(n=20)$. cTnI, cardiac troponin I; AMI, acute myocardial infarction.

$0.68(\mathrm{P}=0.151)$ and $0.93(\mathrm{P}=0.691)$, respectively. According to the threshold value defined as fold-change $\geq 2.0$ and $\mathrm{P}<0.05$, miR-125b-5p and miR-30d-5p were selected for further investigation.

Clinical characteristics of the study population. A total of 230 patients with ACS and 79 healthy controls were assessed. Baseline characteristics of the ACS patients at admission are provided in Table III. Gender distribution and diastolic blood pressure were similar between the patients and the healthy controls. However, compared with healthy controls, patients with AMI and UAP were older, were more likely to be smokers, also suffered from hypertension, diabetes and arrhythmia or had elevated blood glucose, lipid, and uric acid levels, impaired renal function, and decreased heart function (all $\mathrm{P}<0.05$ ). In addition, $\mathrm{cTnI}$ levels were higher in patients with AMI and UAP compared with healthy controls $(\mathrm{P}<0.001)$.

Plasma miR-125b-5p and miR-30d-5p levels in AMI, UAP and healthy control groups. Plasma levels of miR-125b-5p and miR-30d-5p were higher in patients with AMI compared with healthy controls (all $\mathrm{P}<0.001$; Fig. 5). Furthermore, levels of miR-125b-5p and miR-30d-5p in plasma varied among the following sub-groups: miR-125b-5p levels in the STEMI, NSTEMI and UAP groups were 3.73, 4.68 and 1.02, while miR-30d-5p levels were 9.82, 8.29 and 0.95 -fold higher than in healthy controls, respectively (Fig. 5).

Changes of miR-125b-5p, miR-30d-5p and cTnI levels at different time points following chest-pain onset in AMI patients. As presented in Fig. 6, miR-125b-5p and miR-30d-5p were detected in AMI patients as early as $3 \mathrm{~h}$ following chest pain onset. Notably, the expression levels peaked at 3-6 $\mathrm{h}$ and then dropped following $9 \mathrm{~h}$. Meanwhile, cTnI peaked from 6-9 $\mathrm{h}$ and a decrease in levels followed at $12 \mathrm{~h}$.

Specificity and sensitivity of miR-125b-5p and miR-30d-5p as diagnostic biomarkers. ROC analysis was performed to assess whether circulating miR-125b-5p and miR-30d-5p may be used as diagnostic biomarkers for AMI. Fig. 7 and Table IV 


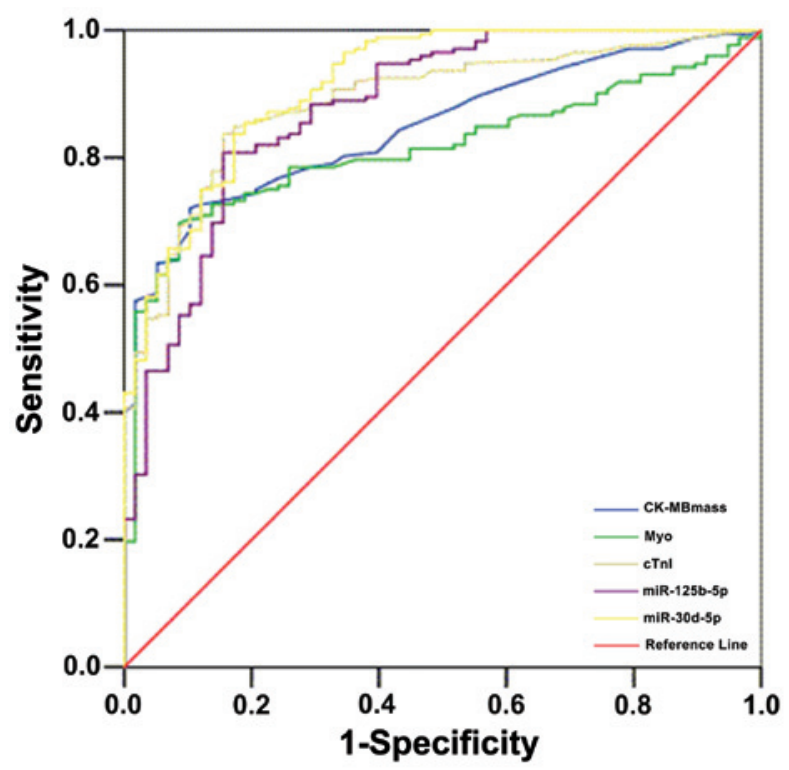

Figure 7. Diagnostic specificity and sensitivity of single biomarker testing in AMI. Receiver operating characteristic of miR-125b-5p, miR-30d-5p, cTnI, Myo, CK-MBmass indicated sensitivity and specificity at admission. CK-MBmass, creatine kinase MB mass; Myo, myoglobin; cTnI, cardiac troponin I; AMI, acute myocardial infarction.

indicates the ROC analysis of CK-MB, Myo and cTnI. The higher area under the curve (AUC) of miR-30d-5p may provide diagnostic information for patients with AMI on admission, with the ability to distinguish AMI from other diseases associated with chest pain. Based on these data, miR-125b-5p is of a similar specificity and sensitivity to $\mathrm{CTnI}$; however, miR-30d-5p exceeded the performance of cTnI. Therefore, miR-125b-5p, and miR-30d-5p may be used to diagnose AMI in patients admitted to the emergency room with symptoms of ACS.

Prognostic value of miR-125b-5p and miR-30d-5p. To further investigate the efficiency of miR-125b-5p and miR-30d-5p as potential biomarkers of AMI, Kaplan-Meier survival analysis was performed for patients with or without AMI. Optimal cut-off values for miR-125b-5p and miR-30d-5p were determined from the corresponding ROC curves to be 2.061 and 2.599 , respectively. Patients were then divided into positive ( $>$ cut-off value) and negative ( $<$ cut-off value) groups. At 6 months, the Kaplan-Meier curve predicted the miR-125b-5p-positive group to have a lower cumulative survival rate than the negative group $(\mathrm{P}=0.045$; Fig. $8 \mathrm{~B})$, but there was no significant difference for miR-30d-5p groups (Fig. 8).

Finally, Cox regression analysis was performed to assess the risk factors involved in the prognosis of patients with ACS. The levels of circulating miR-125b-5p and miR-30d-5p were not significantly associated with the risk of endpoint events at 1, 6 and 12 months (Table V), indicating that they may not reflect the prognosis in patients with ACS.

\section{Discussion}

The aim of the present study was to evaluate circulating microRNAs and their suitability as AMI biomarkers in patients 


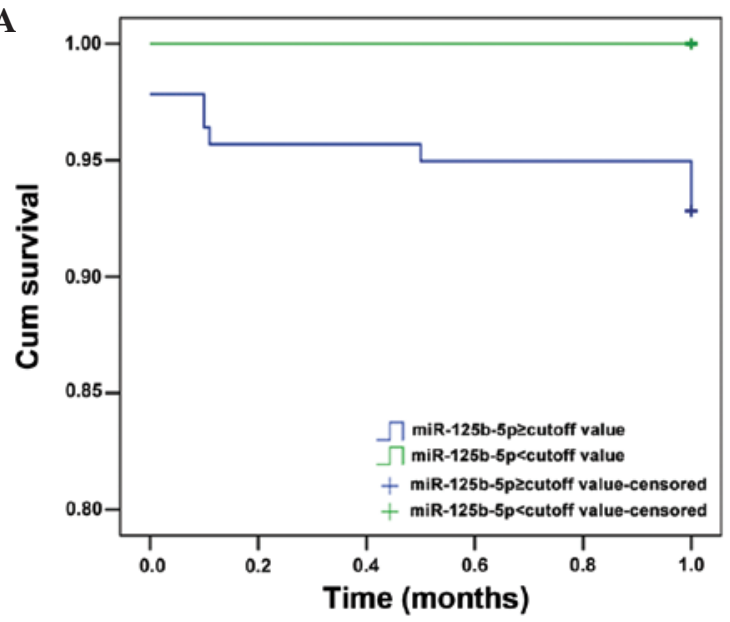

C

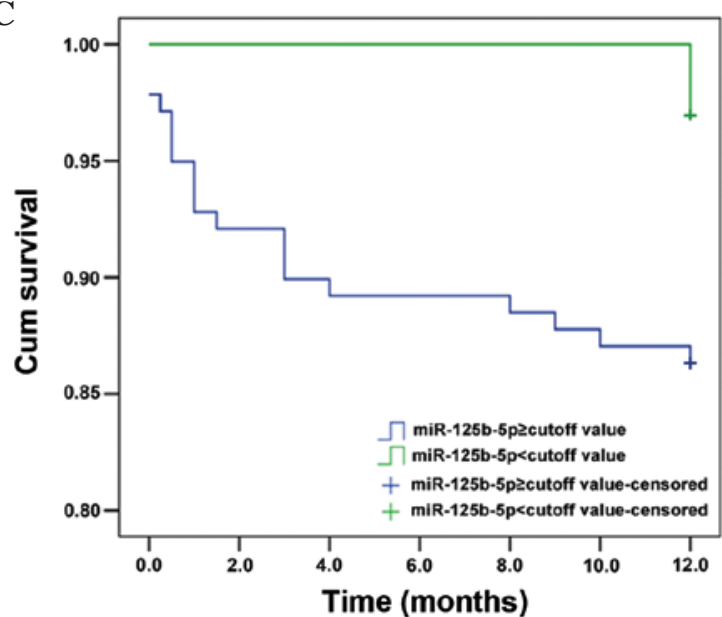

$\mathbf{E}$

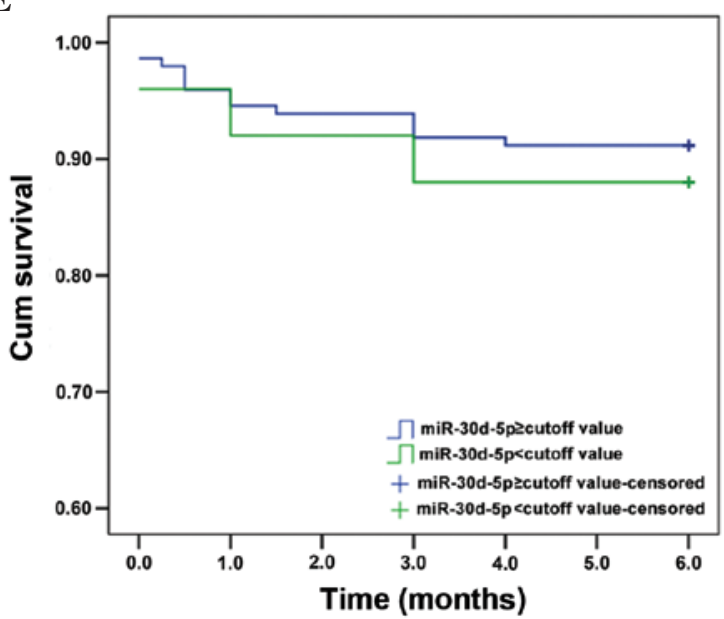

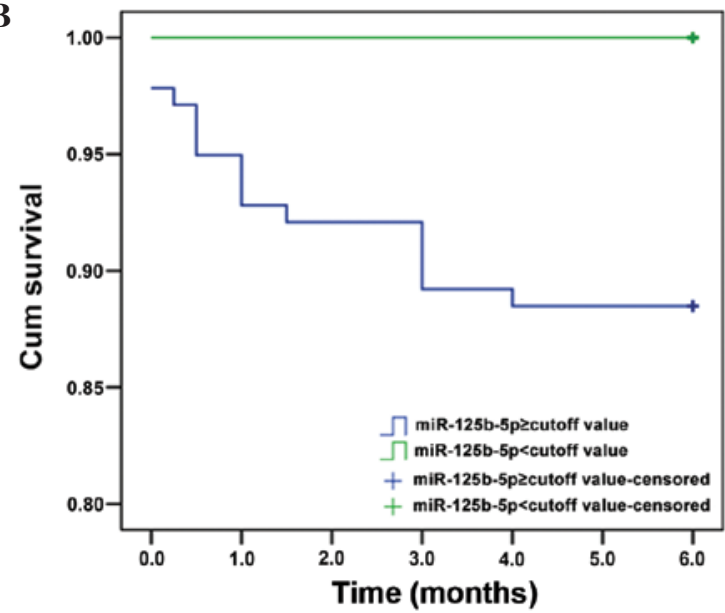

D

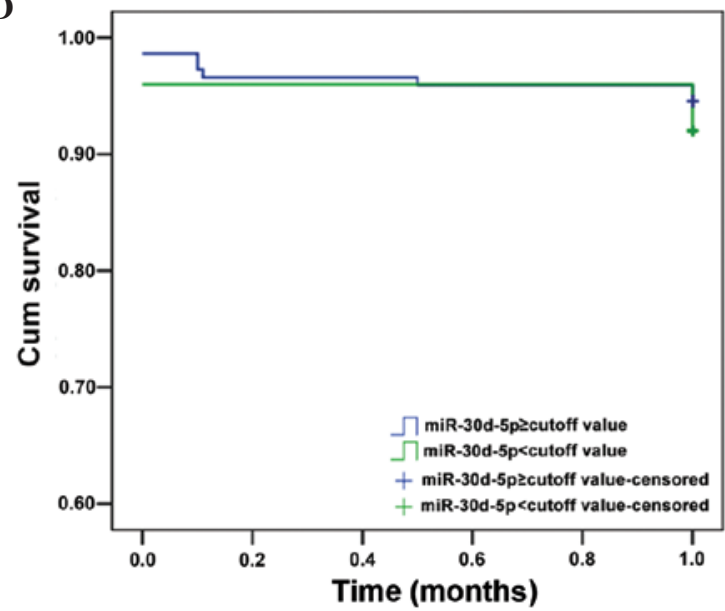

$\mathbf{F}$

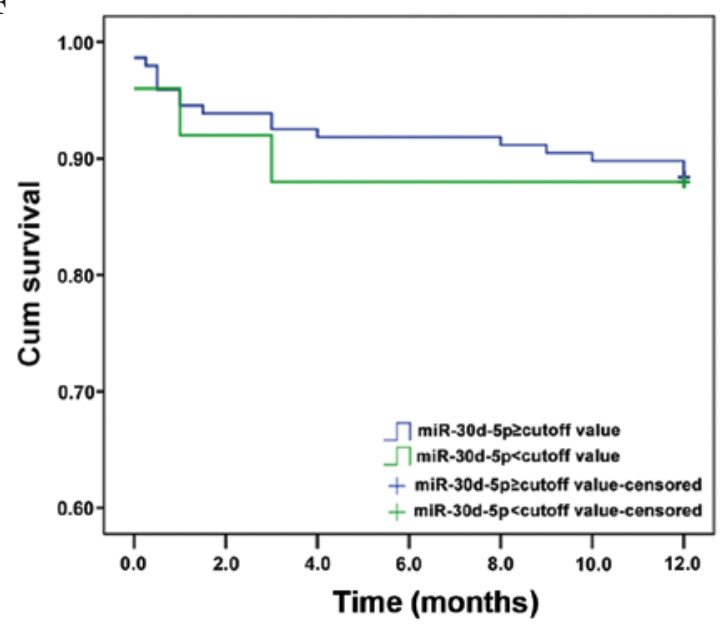

Figure 8. Survival of patients with AMI. Kaplan-Meier curves of cardiovascular events at (A) $1(\mathrm{P}=0.11703)$, (B) 6 ( $\mathrm{P}=0.045)$ and $(\mathrm{C}) 12$ months ( $\mathrm{P}=0.088)$ in 172 patients with AMI, based on miR125b-5p levels at admission (log-rank tests). Kaplan-Meier curves of cardiovascular events at (D) 1 (P=0.616), (E) $6(\mathrm{P}=0.614)$ and $(\mathrm{F}) 12$ months $(\mathrm{P}=0.929)$ in 172 patients with AMI based on miR-30d-5p levels at admission (log-rank tests). AMI, acute myocardial infarction.

with ACS. The results indicated that 33 miRNAs were differentially expressed in patients with AMI and healthy controls. Following validation based on previously published roles for these miRNAs, six miRNAs were validated in an additional five patients and healthy controls. Finally, miR-125b-5p and miR-30d-5p were selected for a more detailed investigation with a larger sample size. Plasma levels of miR-125b-5p and miR-30d-5p were higher in patients with ACS compared with healthy controls (all $\mathrm{P}<0.001)$. ROC curve analysis revealed miR-125b-5p and miR-30d-5p as diagnostic predictors of AMI. Additionally, miR-30d-5p may have a higher diagnostic value than cTnI. Patients with higher levels of miR-125b-5p had poor prognosis compared with those with lower levels.

In the present study, miR-125b-5p, miR-30d-5p, miR-136-5p and miR-129-1-3p were upregulated, while miR-27a-5p and miR-1291 were downregulated. These findings are supported 
Table V. Cox regression analyses of MACE in AMI patients.

\begin{tabular}{lccccccccc}
\hline 1 month & 6 months & 12 months & 95 CI & HR & P & $95 \%$ CI & HR & P & $95 \%$ CI \\
\hline Gender & 1.769 & 0.502 & $0.335-9.333$ & 1.294 & 0.661 & $0.409-4.095$ & 0.801 & 0.664 & $0.295-2.178$ \\
Age & 1.000 & 0.997 & $0.946-1.057$ & 1.030 & 0.221 & $0.982-1.081$ & 1.023 & 0.305 & $0.979-1.069$ \\
cTnI & 0.846 & 0.340 & $0.601-1.192$ & 0.981 & 0.514 & $0.927-1.039$ & 0.996 & 0.834 & $0.961-1.032$ \\
miR-125b & 0.998 & 0.774 & $0.986-1.010$ & 0.999 & 0.855 & $0.991-1.007$ & 0.999 & 0.762 & $0.990-1.007$ \\
miR-30d & 1.000 & 0.834 & $0.997-1.002$ & 1.000 & 0.819 & $0.997-1.002$ & 1.000 & 0.786 & $0.998-1.001$ \\
\hline
\end{tabular}

HR: hazard ratios; 95\%CI: 95\% confidence interval; cTnI: cardiac troponin I.

by previous studies demonstrating the involvement of circulating microRNAs in AMI, including miR-486-3p, miR-150-3p, miR-126-3p, miR-26a-5p, miR-191-5p, miR-133, miR-1291, miR-663b, miR-1, miR-133a, miR-499 and miR-208a (15-18). These miRNAs are associated with muscle tissues in general and more specifically, cardiac muscle. Therefore, they were selected for further validation. miR-125b-5p and miR-30d-5p were selected for further investigation, and their expression was assessed in 230 patients with ACS and 79 healthy controls.

Routine biomarkers for AMI diagnosis include cTnI, CK-MB mass and Myo $(8,9)$. The sensitivity and specificity of miR-125b-5p and miR-30d-5p was then compared with these existing markers. ROC analysis yielded AUC of 0.848, 0.813, 0.889, 0.879 and 0.915, respectively, for CK-MB, Myo, cTnI, miR-125b-5p and miR-30d-5p. The higher AUC of miR-30d-5p and miR-125b-5p may provide diagnostic information for patients with AMI on admission, with the ability to distinguish AMI from other diseases associated with chest pain. Based on these data, miR-125b-5p is of a similar specificity and sensitivity to cTnI; however, miR-30d-5p exceeded the performance of cTnI. This suggests that miR-125b-5p, and miR-30d-5p may be used for AMI diagnosis in patients admitted to the emergency room with symptoms of ACS.

To determine the association between miR-125b-5p, miR-30d-5p and cTnI and ACS onset, these markers were assessed in patients with AMI at different times following the onset of chest pain. Notably, miR-125b-5p and miR-30d-5p were detectable as early as $3 \mathrm{~h}$ after the onset of chest-pain, and peaked at 3-6 h, prior to decreasing at $9 \mathrm{~h}$; meanwhile, cTnI peaked at 6-9 $\mathrm{h}$ and decreased from $12 \mathrm{~h}$. These results strongly suggest that the two miRNAs are released earlier than cTnI. Therefore, these data highlight the potential of the miRNAs as early biomarkers for myocardial injury (25-27). Early diagnosis and treatment of AMI significantly reduces mortality and improves long-term prognosis (6) and the present findings indicate that microRNAs may be superior to cTnI as AMI biomarkers, despite similar AUC, sensitivity and specificity, thus may allow for early diagnosis.

Despite an association between miR-125b-5p and survival using Kaplan-Meier analyses, the present findings indicated that miR-125b-5p and miR-30d-5p were not independently associated with prognosis. The results suggest that miR-125b-5p and miR-30d-5p may be used for the early diagnosis of AMI, but not for prognosis. At present, the prognostic value of miRNAs is limited. To the best of our knowledge, the current study is the first to evaluate the prognostic value of miR-125b-5p in the emergency department for patients with AMI.

Various limitations of the current study should be addressed, including the following: i) This was a single-center study, and the results may not be easily extrapolated to other locations; ii) the kidneys are important in the clearance of various molecules, and may affect the levels of miRNAs, thus making a diagnosis inaccurate; iii) the areas of myocardial injury were not systematically assessed in the patients sampled; and iv) the follow-up period was short, thus there is a possibility that miRNAs may be associated with long-term prognosis. However, further study is required to address this issue.

The possible biological roles of circulatory microRNAs following the onset of chest pain must be further elucidated. However, considering the recent reports describing cell-to-cell transport of miRNAs (28) and data describing miRNAs as paracrine signaling molecules (29), it is possible that circulatory miRNAs are not merely byproducts of myocardial necrosis, thus may also have precise functions. This may point toward a cardioprotective role for these miRNAs in the case of myocardial infarction. These questions should be addressed thoroughly in future studies.

In conclusion, the current study identified that patients with AMI have distinct miRNA profiles compared with healthy controls. In addition, miR-125b-5p and miR-30d-5p may be used as potential early diagnostic biomarkers for AMI; however, their prognostic value may be limited.

\section{Acknowledgements}

The present study was supported by a grant from the Tianjin Natural Science Funds (grant no. 12JCYBJC17400), Tianjin Binhai New Area Health Bureau (grant nos. 2011BHKZ003, 2013BWKY030), and Tianjin Health Bureau (grant no. $12 \mathrm{KG} 139)$.

\section{References}

1. Kumar A and Cannon CP: Acute coronary syndromes: Diagnosis and management, part I. Mayo Clin Proc 84: 917-938, 2009.

2. Anderson JL, Adams CD, Antman EM, Bridges CR, Califf RM, Casey DE Jr, Chavey WE, 2nd, Fesmire FM, Hochman JS, Levin TN, et al: ACC/AHA 2007 guidelines for the management of patients with unstable angina/non-ST-Elevation myocardial infarction. J Am Coll Cardiol 50: e1-e157, 2007. 
3. Jneid H, Anderson JL, Wright RS, Adams CD, Bridges CR, Casey DE Jr, Ettinger SM, Fesmire FM, Ganiats TG, Lincoff AM, et al: 2012 ACCF/AHA focused update of the guideline for the management of patients with unstable angina/Non-ST-elevation myocardial infarction (updating the 2007 guideline and replacing the 2011 focused update. Circulation 126: 875-910, 2012.

4. Amsterdam EA, Wenger NK, Brindis RG, Casey DE Jr, Ganiats G, Holmes DR, Jr, Jaffe AS, Jneid H, Kelly RF, Kontos MC, et al: 2014 AHA/ACC guideline for the management of patients with non-ST-elevation acute coronary syndromes J Am Coll Cardiol 64: e139-228, 2014

5. Braunwald E: Unstable angina and non-ST elevation myocardial infarction. Am J Respir Crit Care Med 185: 924-932, 2012.

6. White HD and Chew DP: Acute myocardial infarction. Lancet 372: 570-584, 2008

7. Chieffo A, Buchanan GL, Mauri F, Mehilli J, Vaquerizo B Moynagh A, Mehran R and Morice MC: ACS and STEMI treatment: Gender-related issues. EuroIntervention 8 (Suppl P): P27-P35, 2012

8. Bellbhudder U and Stanfliet JC: Clinicians ignore best practice guidelines: Prospective audit of cardiac injury marker ordering in patients with chest pain. S Afr Med J 104: 305-306, 2014.

9. Ozturk G, Tavil B, Ozguner M, Ginis Z, Erden G, Tunc B, Azik MF, Uckan D and Delibas N: Evaluation of Cardiac Markers in Children Undergoing Hematopoietic Stem Cell Transplantation. J Clin Lab Anal 29:259-262, 2015.

10. Jaffe AS, Ravkilde J, Roberts R, Naslund U, Apple FS, Galvani M and Katus H: It's time for a change to a troponin standard. Circulation 102: 1216-1220, 2000.

11. Omland T, de Lemos JA, Sabatine MS, Christophi CA, Rice MM, Jablonski KA, Tjora S, Domanski MJ, Gersh BJ, Rouleau JL, et al; Prevention of Events with Angiotensin Converting Enzyme Inhibition (PEACE) Trial Investigators: A sensitive cardiac troponin $\mathrm{T}$ assay in stable coronary artery disease. N Engl J Med 361: 2538-2547, 2009.

12. Devaux Y, Vausort M, Goretti E, Nazarov PV, Azuaje F, Gilson G, Corsten MF, Schroen B, Lair ML, Heymans S and Wagner DR: Use of circulating microRNAs to diagnose acute myocardial infarction. Clin Chem 58: 559-567, 2012.

13. Keller T, Zeller T, Peetz D, Tzikas S, Roth A, Czyz E, Bickel C, Baldus S, Warnholtz A, Fröhlich M, et al: Sensitive troponin I assay in early diagnosis of acute myocardial infarction. N Engl J Med 361: 868-877, 2009.

14. Wang GK, Zhu JQ, Zhang JT, Li Q, Li Y, He J, Qin YW and Jing Q: Circulating microRNA: A novel potential biomarker for early diagnosis of acute myocardial infarction in humans. Eur Heart J 31: 659-666, 2010.

15. Hsu A, Chen SJ, Chang YS, Chen HC and Chu PH: Systemic approach to identify serum microRNAs as potential biomarkers for acute myocardial infarction. BioMed Res Int 2014: 418628, 2014.
16. Peng L, Chun-guang Q, Bei-fang L, Xue-zhi D, Zi-hao W, Yun-fu L, Yan-ping D, Yang-gui L, Wei-guo L, Tian-yong H and Zhen-wen H: Clinical impact of circulating miR-133, miR-1291 and miR-663b in plasma of patients with acute myocardial infarction. Diagn Pathol 9: 89, 2014.

17. Hunter MP, Ismail N, Zhang X, Aguda BD, Lee EJ, Yu L, Xiao T, Schafer J, Lee ML, Schmittgen TD, et al: Detection of microRNA expression in human peripheral blood microvesicles. PLoS One 3: e3694, 2008.

18. Hu SJ, Ren G, Liu JL, Zhao ZA, Yu YS, Su RW, Ma XH, Ni H, Lei $\mathrm{W}$ and Yang ZM: MicroRNA expression and regulation in mouse uterus during embryo implantation. J Biol Chem 283: 23473-23484, 2008.

19. Dweep H and Gretz N: miRWalk2.0: A comprehensive atlas of microRNA-target interactions. Nat Methods 12: 697, 2015.

20. Schmittgen TD and Livak KJ: Analyzing real-time PCR data by the comparative C(T) method. Nat Protoc 3: 1101-1108, 2008

21. FichtlschererS1,DeRosaS,FoxH,SchwietzT,Fischer A,Liebetrau C, Weber M, Hamm CW, Röxe T, Müller-Ardogan M, et al: Circulating microRNAs in patients with coronary artery disease. Circ Res 107: 677-684, 2010.

22. Kroh EM, Parkin RK, Mitchell PS and Tewari M: Analysis of circulating microRNA biomarkers in plasma and serum using quantitative reverse transcription-PCR (qRT-PCR). Methods 50: 298-301, 2010

23. Meder B, Keller A, Vogel B, Haas J, Sedaghat-Hamedani F, Kayvanpour E, Just S, Borries A, Rudloff J, Leidinger P, et al: MicroRNA signatures in total peripheral blood as novel biomarkers for acute myocardial infarction. Basic Res Cardiol 106: 13-23, 2011.

24. Bostjancic E, Zidar N and Glavac D: MicroRNA microarray expression profiling in human myocardial infarction. Dis Markers 27: 255-268, 2009.

25. Iguchi H, Kosaka N and Ochiya T: Secretory microRNAs as a versatile communication tool. Commun Integr Biol 3: 478-481, 2010.

26. Zampetaki A, Willeit P, Drozdov I, Kiechl S and Mayr M: Profiling of circulating microRNAs: From single biomarkers to re-wired networks. Cardiovasc Res 93: 555-562, 2012.

27. Gidlöf O, Andersson P, van der Pals J, Götberg M and Erlinge D: Cardiospecific microRNA plasma levels correlate with troponin and cardiac function in patients with ST elevation myocardial infarction, are selectively dependent on renal elimination, and can be detected in urine samples. Cardiology 118: 217-226, 2011.

28. Valadi H, Ekström K, Bossios A, Sjöstrand M, Lee JJ and Lötvall JO: Exosome-mediated transfer of mRNAs and microRNAs is a novel mechanism of genetic exchange between cells. Nat Cell Biol 9: 654-659, 2007.

29. Zernecke A, Bidzhekov K, Noels H, Shagdarsuren E, Gan L, Denecke B, Hristov M, Köppel T, Jahantigh MN, Lutgens E, et al: Delivery of microRNA-126 by apoptotic bodies induces CXCL12-dependent vascular protection. Sci Signal 2: ra81, 2009. 